\title{
Kaltzeitliche Ablagerungen im Hochland von Angola
}

\author{
Von Frithjof Voss, Hamburg
}

Mit 6 Abbildungen

$\mathrm{Zus}$ a m m e $\mathrm{f}$ assung. Durch Forschungen im Hochland von Angola wurden einige Beweise verschiedenartigen Ursprungs entdeckt, die auf Wirkungen kalter klimatischer Bedingungen in geologisch junger Zeit zurückgehen und wie folgt zusammengefaßt werden können:

1. Die Ausbildung einer früher oberflächenbildenden Steinsohle, wenige Dezimeter unterhalb der heutigen Topographie, scheint sehr weit verbreitet zu sein, sogar über die angegebenen Lokalitäten hinaus.

2. Warventone in der Nähe von Silva Porto entstanden gleichfalls unter ähnlichen klimatischen Einflüssen. Von besonderem Interesse ist jedoch die Diskrepanz zwischen der herkömmlichen Warvenzählung und der Auswertung von Dünnschliffen. Zusätzliche Untersuchungen an anderen Proben werden nötig sein, um das Problem weiter zu verfolgen.

3. Schließlich wurden stark verwulstete, ehemals geschichtete Materialien, Würgeböden, nahe der Oberfläche auf dem „Planalto “ südlich von Nova Lisboa gefunden und makro- sowie mikroskopisch untersucht.

In der vergleichenden Betrachtung der erarbeiteten verschiedenen Fakten ergibt sich aus der vorläufigen Korrelation mit der bis jetzt verfügbaren Chronologie des Quartärs das Haupt Gamblian (30 500-14 503 vor der Gegenwart) als Entstehungszeit.

$\mathrm{S} \mathrm{u} \mathrm{m} \mathrm{mary}$. Researches in the upland of Angola revealed several proofs of various origin, indicating the effects of cool climatic conditions in geologically recent times which can be summarized as follows:

1. A layerlike concentration of stones a few decimeters below the present topography represents a former surface which appears to be extremely widespread, even far beyond the mentioned places.

2. Varve clays in the vicinity of Silva Porto likewise originated under similar climatic influences. Of particular importance are, however, the discrepancies between the data obtained by the application of the conventional field measurements of varves and those derived from the evaluation of thin sections. Further investigations of other samples will be necessary, in order to pursue the problem.

3. Finally, irregularly contorted formerly stratified sediments, involutions, were found near the surface on the "Planalto" south of Nova Lisboa and investigated macro- and microscopically.

Taking the different elaborated facts into consideration, their preliminary correlation with the so far available Quarternary chronology strongly indicates their formation during the Main Gamblian stage (30 $500-14503$ B.P.).

\section{Vorbemerkungen}

Durch das Entgegenkommen von Herrn Prof. Dr. Kolb und mit freundlicher Unterstützung der DFG erhielt ich Ende 1965 die Gelegenheit einer dreimonatigen Forschungsreise durch Angola, für deren Möglichkeit ich mich an dieser Stelle bedanken möchte.

Neben der Bearbeitung eines Hauptthemas, das die feste Bindung an eine bestimmte Reiseroute voraussetzte, ergaben sich vielfältige Ansätze zu weiteren Beobachtungen, von denen eine ausgewählte Anzahl in der vorliegenden Arbeit zusammengefaßt wurde. Auf die Fragestellung des vorliegenden Aufsatzes wurde ich von Herrn Prof. Dr. Borchert hingewiesen, der in seiner Arbeit (Borcher T 1963) bereits ähnliche Phänomene beobachtet hatte, auf die an den entsprechenden Stellen im Text zurückgegriffen wird.

Wegen der bereits beschriebenen Reise- und Arbeitsbedingungen können die folgenden Ergebnisse natürlicherweise nur einen vorläufigen Überblick vermitteln und dürfen nicht als abgeschlossene Darstellung aufgefaßt werden. Es ist sicher, daß die bisherigen Ansätze und Resultate bei einer zukünftigen Spezialisierung des behandelten Fragenkreises beträchtlich erweitert werden können. 


\section{Steinhorizonte}

Nach der bisherigen Literatur über Angola zu urteilen hat nur BorCHERT (1963) das Auftreten von Steinsohlen im Gebiet nordwestlich von Nova Lisboa $\left(13^{\circ} \mathrm{S} / 15^{\circ} \mathrm{E}\right)$ beschrieben. Hier liegen sie zwischen den Erhebungen des Veva, Mocco und Cobongo, überall dort, wo die 1700-1800 m Fläche an den Flanken höherer Berge erhalten ist. In diesen Bereichen folgt unter einer 30-50 cm mächtigen Durchwurzelungszone eine etwa $3 \mathrm{~m}$ umfassende Schicht strukturlosen Rotlehms äolischer Umlagerung, der die talwärts einfallenden, überall einfach auftretenden Steinsohlen aus zugerundeten Quarzitgesteinen überdeckt. Ihnen unterlagern Rotlehme mit noch erkennbarer Struktur des granitischen Ausgangsgesteins in nicht bekannter Mächtigkeit.

Während der eigenen Arbeiten wurden jedoch auch im Dreieck zwischen Quibala, Calulo und Cambambe $\left(10^{\circ} \mathrm{S} / 14^{\circ} 30^{\prime} \mathrm{E}\right)$ sowie östlich von Vila Salazar $\left(9^{\circ} 20^{\prime} \mathrm{S} / 15^{\circ} \mathrm{E}\right)$ Steinhorizonte in tiefer gelegenen Bereichen zwischen 1800 und $750 \mathrm{~m}$ beobachtet.

$\mathrm{Da}$ exakte topographische oder geologische Karten bisher fehlen, lassen sich zum gegenwärtigen Zeitpunkt die Beobachtungen nur in einer Beschreibung zusammenfassen, die allerdings auf Grund der überall ähnlich auftretenden Profile eine gewisse Allgemeingültigkeit besitzt.

Im Gegensatz zum örtlich wechselnden Liegenden der Steinsohlen, das meist aus verschiedenartigen Lehmen unbekannter Mächtigkeit besteht, ist das hervorstechendste Kennzeichen der Horizonte selbst ihr immer und überall e in $\mathrm{f} \mathrm{a} \mathrm{ch}$ es Vorkommen. Sie treten an allen beobachteten Fundorten in Tiefen von $50 \mathrm{~cm}$ bis zu $2 \mathrm{~m}$ unterhalb der heutigen Oberfläche auf und erreichen wechselnde Schichtstärken von $5-30 \mathrm{~cm}$ bei Neigungswinkeln von 2 (Min.) bis 25 (Max.) Grad. In Abhängigkeit von den jeweiligen örtlichen Verhältnissen ließen sich nur in wenigen Gebieten Einblicke in die Verbreitung der Steinsohlen erzielen, jedoch erreichen sie manchenorts gewaltige Ausdehnung. Im größten zusammenhängenden Vorkommen, $24 \mathrm{~km}$ östlich von Vila Salazar, beidseitig der Straße nach Malange, nehmen sie in $750 \mathrm{~m}$ Höhe eine Zone von mindestens 8 ha Fläche ein (Abb. 1). An zahlreichen Einschnitten wiederholt sich $4 \mathrm{~km}$ östlich der beschriebenen Lokalität das gleiche Erscheinungsbild nur in einer etwas geringeren Flächenausdehnung von 5,5 ha gemessener Größe.

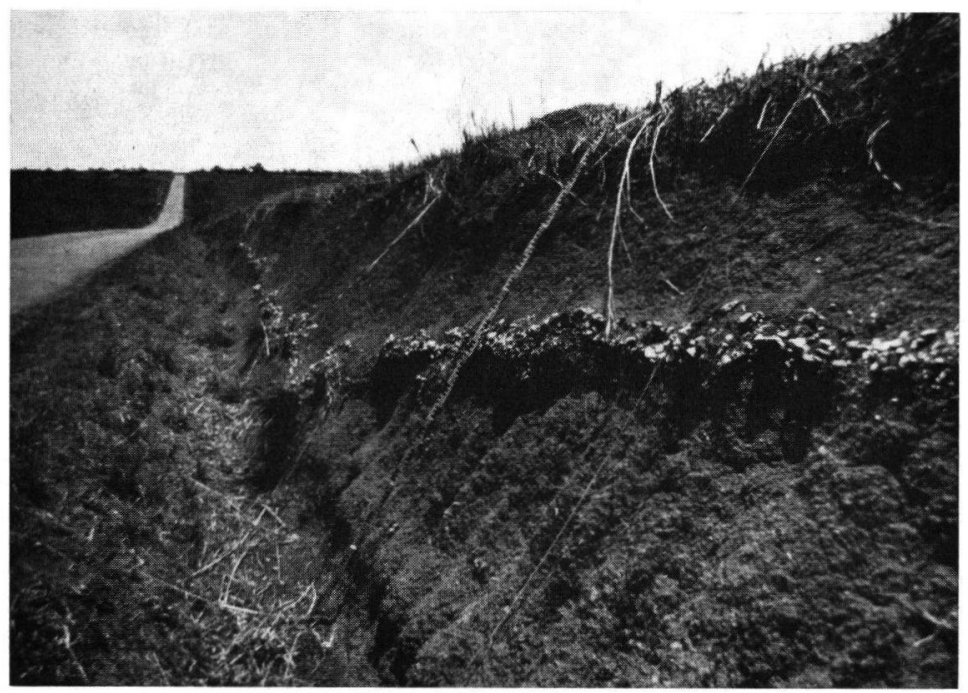

Abb. 1. Steinhorizont $24 \mathrm{~km}$ östlich von Vila Salazar $\left(9^{\circ} 20^{\prime} \mathrm{S} / 15^{\circ} \mathrm{E}\right)$ in einer Höhenlage von $750 \mathrm{~m}$; Länge der auf dem Photo sichtbaren Steinsohle: $350 \mathrm{~m}$; Maßstableiste: $3 \mathrm{~m}$. 
Weitere generelle Merkmale sind in allen Aufschlüssen die eindeutigen Zusammenhänge zwischen Steinhorizont und Untergrund, wobei das Anstehende sich sowohl in einem nicht sichtbaren Relief unter der heutigen Oberfläche befinden kann oder aber meist in unmittelbarer Nähe zu größeren Höhen aufsteigt. Gleichfalls verbunden mit dieser Beziehung sind die sich entsprechenden Gesteinsarten, die eine nicht allzu große Distanz der transportierten Materialien belegen, wie sie darüber hinaus weit besser aus deren $\mathrm{Zu}$ rundungsgrad hervorgeht. An allen Fundorten weisen die der Horizontlage gemäß eingeregelten, in Durchmessern von 2 bis $25 \mathrm{~cm}$ auftretenden Steine scharfe Formen auf, deren Kanten nur in den wenigsten Fällen gebrochen sind. Auch die vielfach andernorts beobachteten Verwitterungserscheinungen an Gesteinen in schalen- oder rindenartiger Weise bilden eine Seltenheit.

Schließlich bleibt noch als auffällige Tatsache erwähnenswert, daß sich in keiner der untersuchten Steinsohlen irgendwelche Einschlüsse fanden, die eine nähere Klärung der Datierung und der Morphogenese jener Schichten hätten bringen können.

Wie bei den Steinhorizonten so lassen sich vorerst ebenfalls für deren Deckschichten die typischen Eigenarten in einer kurzen, überall gültigen Beschreibung zusammenfassen. Sie bestehen in jedem Untersuchungsbeispiel aus umgelagerten Materialien vorwiegender Sand- und Kieskorngrößen der bereits genannten Mächtigkeit mit nur gelegentlich auftretenden Steinen und Blöcken. In nahezu keinem der vielen Fälle war eine eindeutige Schichtung festzustellen; auch läßt sich aus dem makroskopisch sichtbaren Bild nicht die Entstehungsweise der Umlagerung ableiten. Es scheint allerdings im Vergleich mit den gegenwärtigen Vorgängen in den jüngsten Teilen der Deckschichten vieles dafür zu sprechen, daß die Verlagerung der einzelnen Bestandteile überwiegend durch oberflächlich abfließende Wassermengen geschah und geschieht und weniger auf äolische Einflüsse zurückzuführen ist.

Obwohl diese Befunde mit den von FöLster (1964) beschriebenen Pedimentationsprozessen aus dem Südsudan prinzipielle Übereinstimmungen zeigen, fehlt es doch an hinreichend detaillierten Vergleichsmöglichkeiten. Ohne ähnliche Spezialuntersuchungen und großräumige Kartierungen lassen die dargelegten Beobachtungen nur die Schlußfolgerung $\mathrm{zu}, \mathrm{daß}$ es sich bei den Steinsohlen in ihrer Verbreitung und ihren Lagerungsverhältnissen unzweifelhaft an jedem Beobachtungspunkt um Teile einer ehemaligen Oberfläche handelt. Die umrissene Einheitlichkeit und die Art ihres Auftretens an verschiedenen Orten legen zusätzlich ihre etwa gleichzeitige Entstehung nahe, die darüber hinaus auf Grund aller Befunde ein relativ geringes Alter der Steinhorizonte einschließt. Eine allein auf den vorliegenden Untersuchungen fußende Datierung ist im Augenblick wegen fehlender, dafür geeigneter Ablagerungen unmöglich; verläßlich dagegen ist die Aussage zu erschließen, daß sich die Steinpflaster als Oberflächen nur in einer Zeit weit geringerer Vegetationsbedekkung als heute entwickeln konnten. Die sich damit verbindende Frage nach den derzeitigen Klimaverhältnissen ist ebenfalls aus den Untersuchungsbeispielen nicht abzuleiten; eine Antwort darauf läßt sich nur in der abschließenden Zusammenfassung finden.

\section{Warventone}

Die weithin zu beobachtende Verbreitung der Steinhorizonte und die daran anknüpfenden klimatischen Erklärungsmöglichkeiten gaben den Anlaß zur Suche zusätzlicher Kennzeichen, um eine nähere Bestimmung ihrer einstigen Entstehungsvorgänge erzielen zu können. In diesem Rahmen verdiente eine bereits von Borges \& MouTA (1929) beschriebene, von JESSEN (1934) als Glazialvorkommen bezeichnete Ablagerung unweit nordwestlich von Silva Porto $\left(12^{\circ} 30^{\prime} \mathrm{S} / 17^{\circ} \mathrm{E}\right)$ Beachtung, die auch in den vorliegenden $\mathrm{Zu}-$ sammenhängen aufgesucht wurde (Abb.2). Ergänzend zu dem damaligen Bericht stammen aus diesem Bereich in der jüngsten Literatur vier Kurzprofilbeschreibungen von 
BORCHERT (1963), die sich auf bessere zusätzliche Aufschlüsse als vor einigen Jahrzehnten stützen.

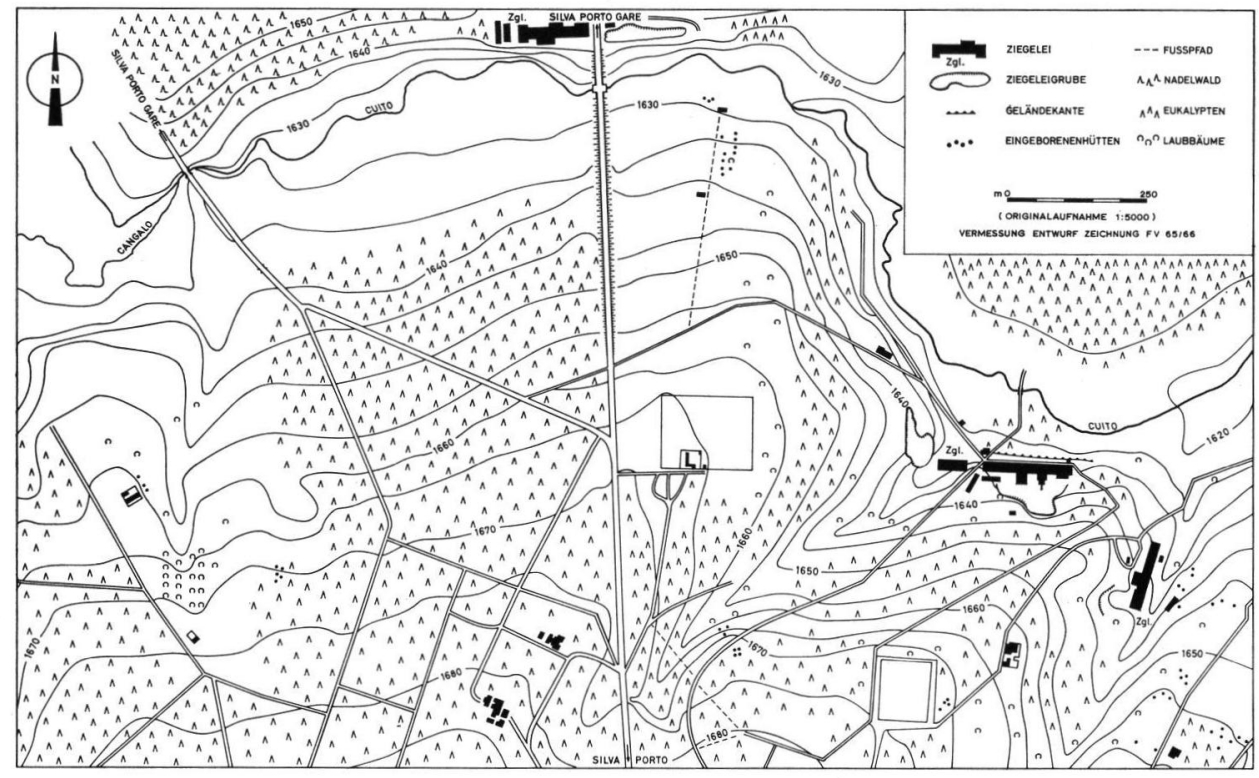

Abb. 2. Das Tal des Cuito zwischen Silva Porto und Silva Porto Gare.

Besonders problematisch ist allerdings die verschiedenartige Bestimmung des angesprochenen Materials, das sich nach Jessen (1934) 30 bis $40 \mathrm{~m}$ hangaufwärts an den Oberflächen beider Talhangseiten als eine blockreiche, sandig tonige Grundmoräne findet. Während diese Schicht im überwiegenden Teil der Abb. 2 kaum hervorsticht, tritt sie auffallend typisch nur zwischen den beiden Straßenführungen von Silva Porto nach Silva Porto Gare am bewaldeten Nordhang des Cuito Tales in Erscheinung. Hier ist nach Jessen (1934) die oberste Zone der Ablagerung durch starke Verwitterungserscheinungen und eine gewisse konglomeratische Verbackenheit gekennzeichnet, die sich mit zunehmender Tiefe in einen auffällig frischen Zustand der Schicht verändert, deren Geschiebezusammensetzung vorwiegend aus Gneisen, Quarziten und Quarzen besteht. Von den gleichfalls angegebenen Beimengungen erratischer Blöcke in Form von dunklen basischen Gesteinen und Graniten ließen sich nur für letztere Bestätigungen finden, während gekritzte Geschiebe ebenfalls nicht entdeckt werden konnten. Mit Recht haben Borges \& Mouta (1929) auf Grund dieser Befunde bereits 1929 den glazialen Ursprung dieses Vorkommens bezweifelt und es mit der Bezeichnung "Conglomérat du Bié" belegt, da sich keine absolut eindeutigen Beweise für eine eiszeitliche Entstehung finden lassen. Vor allem ist es beim augenblicklichen Stand des Wissens ohne großmaßstäbige geologische Kartierungen unmöglich, eine Entscheidung über die Herkunft der sogenannten erratischen Blöcke zu erzielen.

Ubereinstimmender läßt sich dagegen nach rein äußerlichen Gesichtspunkten betrachtet das Vorkommen von Bändertonen beurteilen, die nach Jessen (1934) im Bachbett des Cuito unter der sogenannten Moräne angeschnitten werden. Sie liegen hier in $1637 \mathrm{~m}$ Höhe in nur Quadratmetergröße umfassendem Austritt, direkt nördlich der alten Straßenbrücke über den Rio Cuito, an der ehemaligen Wegführung von Silva Porto nach Silva Porto Gare, die heute durch eine geradlinige Trasse zwischen beiden Orten verkürzt worden ist (Abb. 2). Da diese interessierenden Ablagerungen auch an anderen Stellen der 
Talung auftreten, erwies sich für weitere Untersuchungen die Erstellung einer genauen topographischen Grundlage als unerläßlich. Aus diesen Gründen wurde zunächst eine Karte des betreffenden Geländes im Maßstab 1 : 5000 mit terrestrisch tachymetrischen Methoden aufgenommen, als deren Ausgangsbasis sich ein dreieinhalb Kilometer nordnordöstlich des Arbeitsbereiches gelegener Höhenpunkt $(1737,17 \mathrm{~m})$ anbot. Die so entstandene kartographische Darstellung enthält außer der Topographie vor allem sämtliche in Frage kommenden Aufschlüsse, die zugleich auch Ziegeleigruben sind; eine Ausnahme bildet in dieser Reihe nur das bereits beschriebene, von JESSEN (1934) erwähnte Vorkommen.

In einem Gesamtüberblick zeigt sich in der Lage und Verteilung der Warventone, daß sie in jedem Fall in ihrer vollständigen, meist mit der Größe der Gruben identischen Ausdehnung an die Talformen des Cuito gebunden sind. Dieser Beweis ließ sich durch weitere Geländearbeiten in Form von Bohrungen und Aufgrabungen in den Randbereichen der

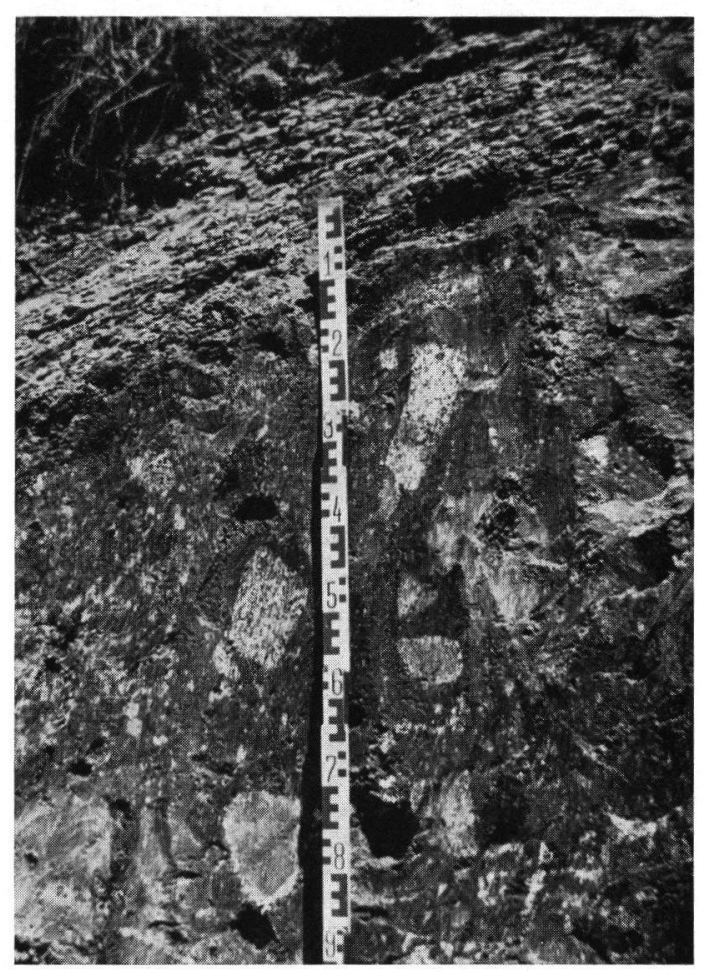

Abb. 3. Typische Aufgrabung der ausdünnenden Warven an den Rändern der Ziegeleigruben nördlich von Silva Porto (Abb. 2). Das Bild zeigt die allgemeingültigen Verhältnisse in allen Aufschlüssen. Die Schräglage der Bändertone ist durch Rutschungen hervorgerufen worden.

Ablagerungen erbringen (Abb. 3), die eindeutig überall ein Ausdünnen der Warvenmächtigkeit in aufwärtiger Talhangrichtung und ihr Austreten an der Oberfläche beweisen. Mit Ausnahme des von Jessen (1934) entdeckten Aufschlusses und der westlichen Grube in der mittleren der drei Ziegeleien finden sich nirgends auflagernde jüngere, umgelagerte Materialien. Auch die untersuchten Schichtmächtigkeiten der Bändertone sprechen deutlich für ihre Lagebeziehung innerhalb der vermessenen Talformen, wie sie zum Beispiel an der östlichen Materialentnahmestelle der zentral gelegenen Ziegelei durch Bohrungen nachgewiesen werden konnten. Während hier die Grenzschichten bei $1631 \mathrm{~m}$ enden, ist in der $1 \mathrm{~km}$ entfernten nordwestlich gegenüberliegenden Grube an der Straße nach Silva Porto 
Gare die Sohle schon bei $1635 \mathrm{~m}$ erreicht. Ähnliche Ergebnisse wurden in dem am östlichen Ortsausgang von Silva Porto gelegenen Ziegeleibetrieb gewonnen, wo die Warvenablagerungen in einer Höhe von etwa $1638 \mathrm{~m}$ angetroffen wurden. Vergleichbare Zusammenhänge ergaben sich auch aus den Feststellungen und Messungen der höchstgelegenen Warvenabsätze an den verschiedenen Stellen im Cuito Tal, die um geringe Meterbeträge schwanken. Beispielsweise erreichen die gebänderten Tone im Westen der Abb. 2, nördlich der Straßenbrücke, ihre größte Höhenlage bei $1637 \mathrm{~m}, 750 \mathrm{~m}$ nordöstlich davon wurden in der Ziegeleigrube an der Hauptstraße $1642 \mathrm{~m}$ auf die längste Distanz ihrer Ausdehnung gemessen. Auf der Südseite des Cuito Tales lagern die Warven an der jüngsten Materialentnahmestelle der zentral gelegenen Ziegelei in Hangnähe bis zu $1642 \mathrm{~m}$ Höhe, während sie in der Grube südlich des Ziegeleigebäudes bei $1638 \mathrm{~m}$ aufhören. Den größten Vergleichswert mit $1644 \mathrm{~m}$ ergaben schließlich die Vermessungen in dem am weitesten östlich gelegenen Aufschluß, der sich in ein kleines namenloses Nebental des Cuito hineinzieht (Abb. 2).

Die auffällige Unterschiedlichkeit von Lagerung und Höhenlage der Bändertonansätze hat keinen Zusammenhang mit einem somalischen Streichen der Warven, das JesseN (1934) seinerzeit in seinem Profil festgestellt hatte. Vielmehr ist das in jedem Aufschluß wechselnde Streichen und Fallen der Schichten zusammen mit dem unterschiedlichen Höhenansatz nur mit ihrer speziellen Lage innerhalb des Cuito Tales zu erklären. Diese Eigentümlichkeiten im Schichtenaufbau sind überall dort $\mathrm{zu}$ beobachten, wo durch die Seiten- und Tiefenerosion des Flusses die Hangform angeschnitten und der Talboden tiefer gelegt wurde, so daß sich im Gefolge dieser Vorgänge bei Durchfeuchtung infolge nahen Grundwasserstandes Rutschungen durch Schwerkrafttransport ereigneten. Ihre Auswirkungen lassen sich sowohl im kleintektonischen Bild der Aufschlüsse als auch besonders eindrucksvoll auf den Oberflächen der Hänge beobachten, wo sie oft viele Meter von den Abbaustellen entfernt in langen talparallelen Spalten und Rissen in Erscheinung treten. Die einzige Ausnahme völlig störungsfrei lagernder Warventone zeigte sich auf dem Gelände der zentral gelegenen Ziegelei in der westlichen Grube, an deren beiden gegenüberliegenden $80 \mathrm{~m}$ langen und $5 \mathrm{~m}$ hohen Abbauwänden keine Veränderungen in der Horizontallage der Warven festgestellt werden konnten. Dieser Befund bestätigt zugleich die Richtigkeit der vorhergehenden Ableitungen, da die Grubenwände keilförmig quer in einen nach Osten vorspringenden, weit vom Haupttal entfernten Hangsporn vorgetrieben wurden (Abb. 2), in dessen tieferen Teilen kaum Feuchtigkeitsansammlungen wegen der Entwässerung eines kleinen Nebenbaches auftreten können.

Die durch die morphologischen Untersuchungen mehrfach bestätigte Abhängigkeit der Bändertonlagen vom Talformenschatz des Rio Cuito findet eine weitere Einengung in den Beziehungen der Warvensedimente zum Liegenden. Soweit es die Anzahl aller Aufschlüsse zeigte, bestehen die unterlagernden Schichten überall aus tiefgründig zersetzten braunroten Lehmen unbekannter Ausdehnung mit mehr oder minder häufig eingelagerten, verwitterten Blöcken unterschiedlicher Größe. Diese meist leicht mit dem Spaten zu durchstechenden Gesteine überwiegend gneisischer Struktur und noch teilweise im Kern erhaltenem Mineralbestand fanden sich auch gelegentlich innerhalb der frisch aussehenden, überall unverwitterten Warventone, in die sie nur durch Rutschungsvorgänge hineingelangt sein können.

Wesentlich deutlicher jedoch waren die Zusammenhänge zwischen den Bändertonen und dem Liegenden durch mikroskopische Untersuchungen im Gelände feststellbar, die das Auftreten gleicher Mineralien aus den verwitterten Gesteinen als umgelagerte Partikel in einzelnen, meist hell bis gelb gefärbten Warven belegten. Waren in diesen Lagen überwiegend Quarze mit maximalen Korngrößen bis 0,1 mm und seltene Glimmerpartikel anzutreffen, so bestanden die dazwischen geschalteten roten Bänder aus feinsten Tonen, die nur dem gleichgefärbten lehmigen Grundmaterial des Liegenden entstammen können. 
Entsprechend bestätigende Ergebnisse erbrachten die Untersuchungen ungestörter Proben im Dünnschliff (Abb. 4), auch wenn deren Resultate allerdings zugleich die durchgeführten Warvenzählungen am ungestörten Profil der Westgrube der zentral gelegenen Ziegelei in Frage stellten. Denn sowohl im feuchten Zustand an der Aufschlußwand als auch in getrockneter Probenform konnten die Feinheiten der im Dünnschliff (Abb. 4) sichtbaren Warvengliederung nicht bemerkt werden, so daß sich erhebliche Auszählungsfehler ergaben. Die Gegenüberstellung der beiden verschiedenen Arbeitsweisen (Abb. 4) belegt zugleich auch die Unmöglichkeit einer sicheren Interpretation der Bänderung und zeigt eindeutig die Notwendigkeit der Verwendung ganzer Reihen von Dünnschliffen für eine exakte geochronologische Auswertung. Inwieweit dieses Ergebnis auch für andere Warvenzählungen gültig ist, läßt sich im Augenblick auf Grund fehlender Vergleichsuntersuchungen nicht abschätzen und muß weiteren Prüfungen vorbehalten bleiben.

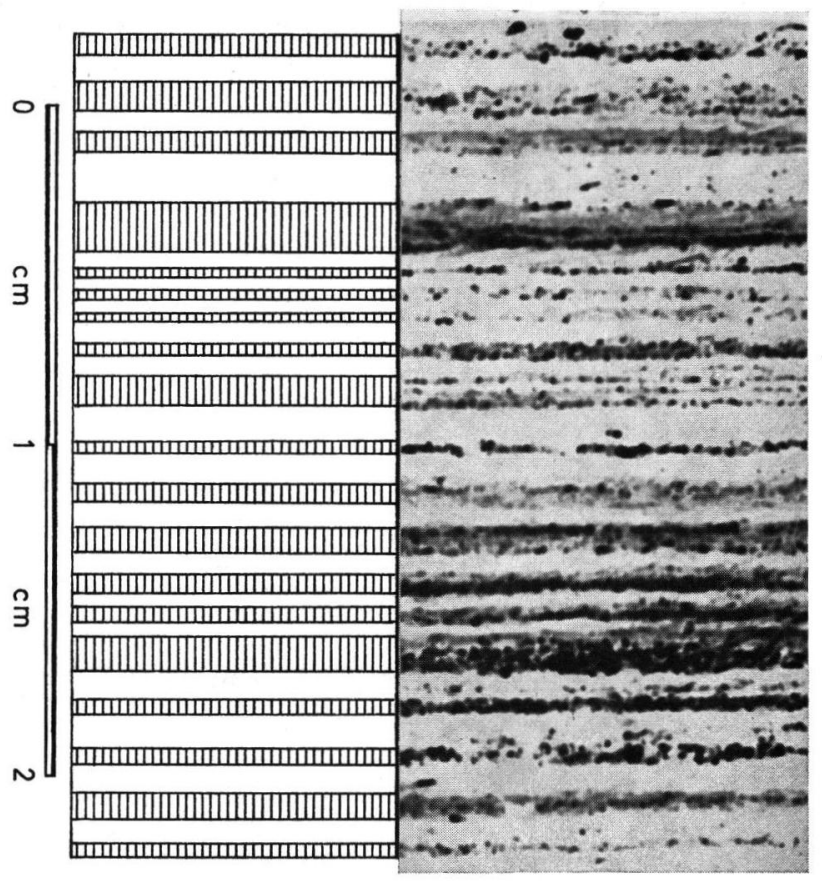

A

B

Abb. 4. Gegenüberstellung der Ergebnisse von Warvenzählungen im Aufschluß (A) mit entsprechenden Resultaten des gleichen Beispiels im Dünnschliff (B). Die unterschiedliche Auszählung beruht auf der makroskopisch nicht sichtbaren Feingliederung der Bändertone. Der Vergleich beider Arbeitsweisen zeigt deutlich die Notwendigkeit ganzer Schliffreihen für gesicherte, zuverlässige geochronologische Bestimmungen. Die waagerechten hellen Partien werden von feinsten Tonen gebildet, die dazwischen liegenden langgestreckten oder runden schwarzen Flecken bestehen aus locker gelagerten Quarzen und umgebenden Hohlräumen.

Die abschließende Frage nach der Ablagerungsdauer der Bändertone bleibt damit also offen, gleichwie eine präzise Altersdatierung der Warvenabsätze nicht möglich war, da weder bei der jahrelangen Abbautätigkeit noch bei den Geländearbeiten mit dem Mikroskop die geringsten Einschlüsse gefunden wurden. Die Annahme einer permokarbonischen 
Stellung von glazigenen Ablagerungen im Cuito Tal nach Jessen (1934) und MoutA (1954) trifft jedoch für die Warventone auf Grund der eigenen Arbeitsresultate nicht zu. Relativ gesehen deuten die Ergebnisse der morphologischen Untersuchungen auf eine junge Entstehungszeit dieser Absätze hin, die sich aus der abschließenden Zusammenfassung einzelner Fakten ableiten läßt.

Der sicherste Ausgangspunkt ist in dieser Beziehung der mehrfache Nachweis einer Bindung an bestimmte beschriebene Ausbildungen des Liegenden und an bereits vorhandene Hohlformen innerhalb des jetzigen Cuito Tales. Allerdings ist die Herkunft und Morphogenese jener vorgezeichneten Senken im Rahmen des eng regional begrenzten Arbeitsgebietes nicht zu klären; außerdem fehlt es bisher an entsprechenden Untersuchungen, die irgendwelche Vergleiche ermöglichen würden. Mit Sicherheit läßt sich nur das Vorhandensein mehrerer voneinander getrennter Becken auf Grund der gemessenen unterschiedlichen Höhenansätze der Warven in den einzelnen Gruben erschließen, deren Werte auch durch die mancherorts beobachteten geringen Rutschungen nicht namhaft beeinträchtigt werden. Selbst wenn die einstigen Ausdehnungen der Senken in der morphologischen Uranlage des Cuito Laufes wegen der gezeigten späteren tiefen- und seitenerosiven Vorgänge nicht mehr völlig rekonstruierbar sind, so läßt sich doch für die Warvenentwicklung eine maximal mögliche Aufhöhung bis zu den niedrigst gelegenen Überlaufpunkten ihrer Beckenumrandungen folgern. Damit ist jedoch nicht gesagt und nicht zu beweisen, daß alle einstigen Gewässer während der Sedimentationsvorgänge miteinander in Beziehung standen.

Mit Sicherheit ist diese morphogenetische Entwicklungsphase der Verbindung aller einstigen Senkenzonen erst in geologisch jüngster Zeit durch die schnelle rückschreitende Erosion erfolgt, deren Auswirkungen auf die Zerstörung der $1700 \mathrm{~m}$ Hochfläche gerichtet ist (Abb. 2). Relikte aus jener Zeit sind als Terrassenreste mit geringer weißer Flußsandbedeckung am Westrand der Abb. 2 nachweisbar, wo sie $350 \mathrm{~m}$ südlich der Einmündung des Cangalo in den Cuito in 1650-1655 m Höhe liegen. Des weiteren werden Oberflächenteile des Eukalyptenwaldes im Nordosten zwischen 1625 und $1630 \mathrm{~m}$ von fluviatilen Sanden eingenommen, die sich schließlich ebenfalls in gleicher Höhenlage im rechten Abbildungsteil, $400 \mathrm{~m}$ östlich der beiden eng benachbarten Ziegeleien, auf einem zum Cuito nordwärts vorspringenden Terrassensporn wiederfinden. Aus diesen bisher nur lokal beobachteten Erscheinungen lassen sich jedoch im Augenblick ohne überregionale Kenntnisse keine weiteren Zusammenhänge ableiten.

Außer den morphologischen Fakten sprechen auch die Ergebnisse der gestörten Lagerungsverhältnisse der Warven und deren Ursachen für eine junge Entstehung der Bändertone. Vor allem belegen nicht feststellbare Verwitterungserscheinungen im Vergleich mit umgebenden Bereichen diese Schlüsse, die sowohl durch die mikroskopischen Untersuchungen von Proben im Gelände als auch durch den exemplarischen Beweis der Abb. 4 gestützt werden. Zusätzlich läßt sich aus diesen ausgewählten Beispielen eine weitere allgemeingültige Ableitung für ein relativ geringes Alter der Ablagerungen erzielen, die abschließend zugleich auch alle vorherigen Behauptungen unterbaut. Eindeutig wird nämlich durch die Anordnung langgestreckter Hohlräume im Sediment die denkbare Möglichkeit einstmals überlagernder Deckschichten ausgeschlossen, wie sie für den $85 \mathrm{~km}$ nordnordwestlich Silva Portos gelegenen Raum von Canata postpermisch in mindestens $200 \mathrm{~m}$ Mächtigkeit nachgewiesen wurden (BORCHERT 1963). Sprechen schließlich alle vorhergehenden Befunde für eine relativ kurz zurückliegende Entstehungszeit der Warventone, so lassen sich die klimatischen Eigenarten während ihrer Ablagerung auf Grund fehlender Fossilien zumindest als Kennzeichen einer sehr vegetationsarmen Periode charakterisieren. In Verbindung mit den zweifellos lange vorhanden gewesenen Wasserüberschüssen während der Sedimentationsvorgänge würde sich aus den gesammelten Tatsachen nur eine kalt feuchte Epoche als äußere Voraussetzung der Bändertonentwicklung erschließen lassen. 

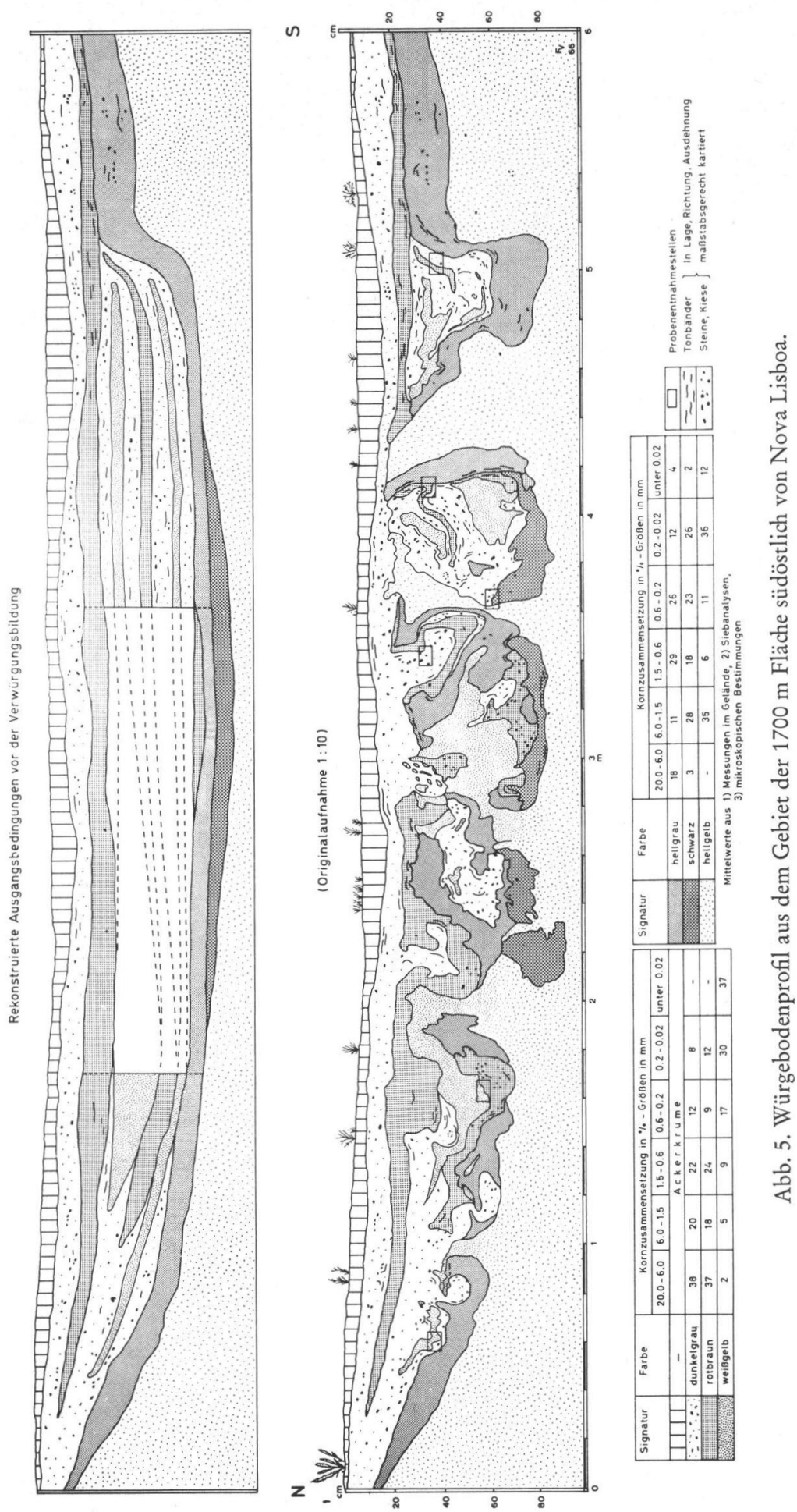


\section{Würgeböden}

Anläßlich einer Geländebegehung im Randbereich des $1700 \mathrm{~m}$ hoch gelegenen Planaltos wurde auf der nahezu ebenen Hochfläche, $2 \mathrm{~km}$ südlich von Nova Lisboa $\left(13^{\circ} \mathrm{S} / 16^{\circ} \mathrm{E}\right)$, in einem Eingeborenenfeld ein Bodeneinschlag von $30 \mathrm{~cm}$ Durchmesser und $30 \mathrm{~cm}$ Tiefe mit deutlichen Verfärbungen entdeckt. Weitere Nachprüfungen legten gut sichtbare $\mathrm{Ma}-$ terialsortierungen in Form von schräggestellten Schichten frei und veranlaßten größere Aufgrabungen an verschiedenen Stellen, aus deren Verbindung untereinander schließlich der auf der Abb. 5 sichtbare Aufschluß hervorging. Bereits während dieser Arbeiten widerlegten entstehende Abschnitte birnenförmig in den Boden sich einstülpender Schichtenverwulstungen die Vermutung, daß es sich bei den vorliegenden Bildungen um Folgeerscheinungen des Wanderhackbaues handeln könnte. Vielmehr zeigte sich auf Grund auffallender äußerer Ähnlichkeiten der gekröseartigen Strukturen mit solchen höherer Breiten die Notwendigkeit, genaue Aufnahmen und Untersuchungen des Profils anzustellen. Aus diesen Gründen wurde bereits während der Fertigstellung des Nord-Süd ausgerichteten Suchgrabens von $7 \mathrm{~m}$ Länge, 1,3 m Breite und $1 \mathrm{~m}$ Tiefe eine Anzahl von Ergebnissen gesammelt, bevor mit der Anfertigung eines typischen Längsschnittes der geglätteten Aufschlußwand nach archäologischer Manier begonnen werden konnte. Mit der Ausgangsbasis einer waagerecht an der Oberfläche gespannten, mit Dezimetermarken versehenen Meßleine ließen sich in der Horizontalen und Vertikalen alle äußerlich sichtbaren Einzelheiten kartieren und im Maßstab 1:10 auf Millimeterpapier übertragen. Diese Arbeitsweise erlaubte an Ort und Stelle in Verbindung mit den Beobachtungen während der Grabung die genaue Festlegung der Schichtenlagerung, ihrer Grenzen, Mächtigkeiten, unterschiedlichen Färbungen und Korngrößenzusammensetzungen, deren alle noch zeichnerisch darstellbaren Kiese und Steine lage- und maßstabgetreu übertragen wurden (Abb. 5). Vor allem konnten bei der Bestimmung sämtlicher Details in schwierigen Fällen die Entscheidungen durch zusätzliche Probenentnahmen leicht gefällt werden, so daß damit eine verläßliche Genauigkeit erreichbar war.

Aus der Verbindung aller durch makroskopische Untersuchungen gewonnenen Ergebnisse läßt sich zunächst die Ausdehnungsbeschränkung der interessierenden Ablagerungen auf den engen Raum einer ovalen, 6 mal $5 \mathrm{~m}$ messenden einstigen Muldenform unbekannter Entstehungsursache festlegen. Ihre Morphogenese muß als Problem wegen bisher fehlender geomorphologischer Studien über diesen Raum genauso offen bleiben wie dasjenige der Herkunft der verschiedenen, in die Senke eingelagerten Materialien. Die Beantwortung dieser Frage fällt angesichts der Fundstellenlage im Wasserscheidenbereich des hochgelegenen Flächenrestes besonders schwer und ist durch die heutigen Reliefgegebenheiten nicht zu erklären. Zum weitaus überwiegenden Teil handelt es sich bei jenen jüngeren Ablagerungen neben Sanden mit gelegentlich auftretenden Kiesen und Steinen vor allem um einstige Lateritkrustenbestandteile in verschiedenster Form. Diese bestehen einerseits aus rötlich braunen Partikeln von Mittelkies bis Grobsandgröße mit noch deutlich erkennbarer typischer Struktur, schalenartiger Verwitterungsschicht und allseitig zugerundeten Oberflächen. Andererseits finden sich die Zerfallsprodukte dieser Lockergesteine in mannigfacher Ausbildung von zertrümmerten Resten der Kiese und Sande über Quarzteilchen mit noch deutlichen Rotfärbungen in Vertiefungen und Rissen bis hin zu Schluffen und feinsten Tonen (Abb. 6).

Die Besonderheit der verschiedenen beschriebenen Bestandteile ist allerdings ihr nach bestimmten Korngrößen geordnetes Auftreten in Form teils stark voneinander abweichenden Schichten, die in größerer Anzahl in die Muldenform hineinziehen. Während das überall auf der umgebenden Fläche festgestellte Normalprofil von ungestörter horizontaler Lagerung gekennzeichnet ist und dem Anschnitt am Nordrand des Suchgrabens entspricht (Abb.5), verändern sich diese Merkmale spontan mit dem Eintritt in die im Liegenden 

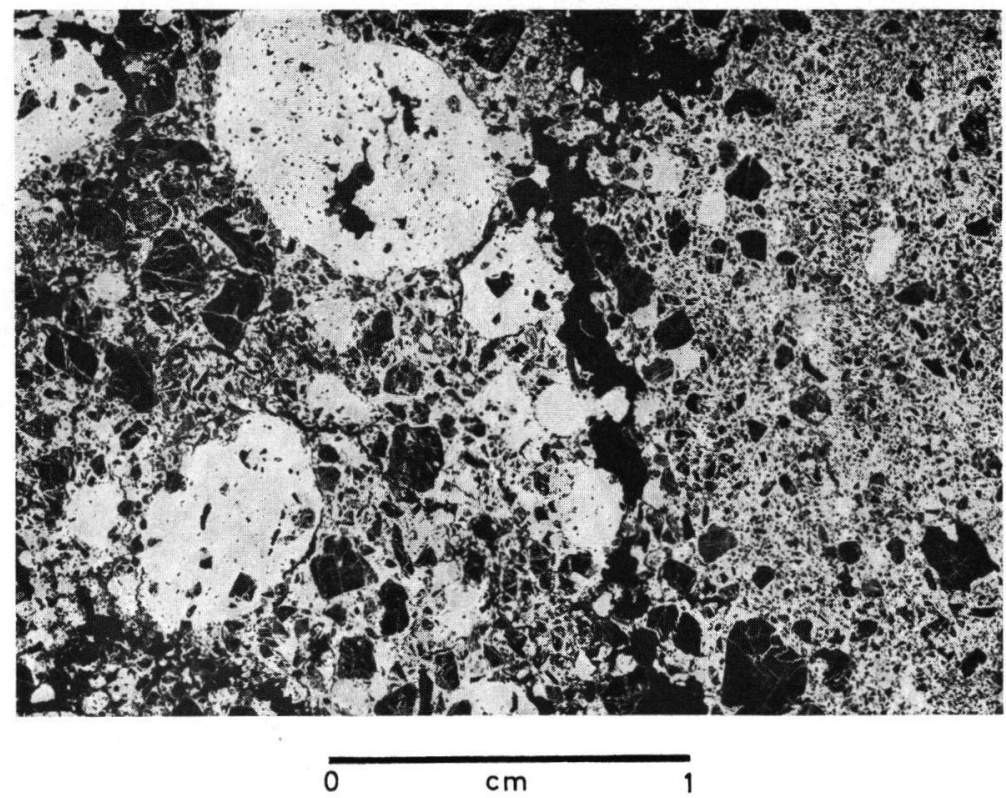

Abb. 6. Dünnschliff der Probe 2 aus dem Profil der Abb. 5 (Lage: $30 \mathrm{~cm}$ unter der Oberfläche und $410 \mathrm{~cm}$ vom Längsschnittbeginn entfernt). Im Vergleich mit der Originalaufnahme, Abb. 5, hebt sich deutlich die Schichtgrenze durch schwarze, langgestreckte Hohlräume und umgebende unterschiedliche Materialzusammensetzungen ab. Alle sichtbaren Bestandteile sind Reste einstiger Lateritkrusten. Besonders auffällig sind die Einregelungserscheinungen der unteren Korngrößen, vornehmlich auf der rechten Seite der Abb. (Erklärung siehe Text.)

vorgezeichnete Senke. Deutlich vermehrt sich vom allseitig umrahmenden Muldenrand her die bekannte Abfolge um wenige nur auf die Senkenform beschränkte Schichten, deren keilförmige Enden in Bodeneinschlägen wie im Nordteil der Aufgrabung in Erscheinung treten (Abb. 5). Besonders bemerkenswert ist jedoch mit zunehmender Annäherung zum Muldenmittelpunkt ein girlandeförmig sich verformendes Liegendes und zugleich ein verstärktes Verwinden aller Bodenschichten, deren Grenzen und Zusammenhänge zur Mitte hin durch Zerreißungen, Quetschungen, Verwürgungen und Injektionen verwischt und vermengt werden. Stellenweise ist sogar ein mauerartiges Aufsteigen des Liegenden bis zur Oberfläche oder seine direkte Verbindung mit durchstoßenden, auf die Muldenform beschränkten Schichten zu beobachten. Auch der Austausch ganzer Schichtfetzen oder ihr wellenförmiges Absinken gehören zu diesen Besonderheiten. Bemerkenswerterweise finden sich jene stattgefundenen, beschriebenen Bewegungsvorgänge auch in der makroskopisch sichtbaren Zusammensetzung der verschiedenen Schichten wieder, in deren Verbiegungsrichtungen stets eine Orientierung der unsortierten Kiese und gelegentlich auftretender dünner Tonbänder vorhanden ist (Abb. 5).

Die Analyse der beobachteten Details während der Grabung und der Kartierung führte schließlich zu einer Rekonstruktionsmöglichkeit der einstigen Lagerungsverhältnisse innerhalb der muldenförmigen Einsenkung, die sich an dem typischen Längsprofil (Abb. 5) nachvollziehen läßt. Unzweifelhaft sicher lassen sich im Nordbereich der Senke neben den bekannten, weit verbreiteten oberflächennahen Schichten die Ansätze derjenigen jüngeren auszählen, die überall im Umkreis nur auf kurze Distanz zur Mitte hin verfolgbar sind. Im Fall der dritten, unterhalb der Feldfläche befindlichen, bereits verbogenen und zerteilten Lage läßt sich sogar ihre einstige Zusammengehörigkeit durch einfache Ausmessung der vorhandenen Reste belegen. 
Diese Kenntnisse sind selbst auf die bereits randlich verformten Partien im Süden des Aufschlusses übertragbar, wo mit ihrer Hilfe die korrespondierende Anzahl der einzelnen Bänder identifizierbar ist. Gemessen an der auftretenden Regelmäßigkeit dieser Schichtenabfolge lassen sich ihre ursprünglich vorhandenen Verbindungen auch für den Mittelteil des Senkenbereichs als ehemals kennzeichnend vermuten, wodurch zugleich eine Zuordnungsprüfung der verwürgten Schichtenteile am Normalfall möglich ist. In Anwendung jener Arbeitsweise lassen sich die Grenzen sicherer Aussagen beträchtlich zur Profilmitte hin ausweiten, so daß schließlich nur ein inneres Drittel der Fläche übrigbleibt, in dessen Bereich sich die Rekonstruktion wegen der ausgeprägten Gekrösestrukturen etwas schwieriger gestaltet. Auch wenn hier Aufgrabungsbeobachtungen unterstützend herangezogen werden können, deren Profilschnitte für den fraglichen Mittelteil nur gelegentlich als sichere Vergleiche verwendbar sind, so ist die einstige Schichtenabfolge trotzdem in befriedigender Weise schematisch ableitbar (Abb. 5 oben).

Damit ist schließlich die Möglichkeit gegeben, an diesem Standardquerschnitt das ungefähre Ausmaß der Verbiegung und Verwürgung oder die Bewegung einzelner Schichtenteile im Boden zu prüfen, auch wenn ihre früheren Mächtigkeiten an keiner Stelle mehr abschätzbar sind.

Zur ursächlichen Erklärung der beschriebenen Turbulenzerscheinungen waren allerdings die im Gelände benutzten Arbeitsweisen angesichts der besonderen geographischen Fundstellenlage nicht vollends ausreichend, so daß zusätzliche Detailuntersuchungen auf die rekonstruierten Ablagerungsverhältnisse ausgedehnt wurden. Insbesondere galten diese der makroskopisch nicht weiter bearbeitbaren Zusammensetzung der unterschiedlichen Schichten, deren ergänzende Korngrößenbestimmungen mit Hilfe des Mikroskops und durch Siebanalysen mitgeführter Proben abgesichert werden konnten (Abb. 5).

Vor allem jedoch erschien eine Untersuchung der beobachteten Orientierung von Kiesen und kleineren Steinen in ihrer Verbreitung innerhalb geringerer Korngrößenzusammensetzungen als ein Weg, die gesuchten Entstehungsursachen näher einzugrenzen. Zu diesem Zweck waren an ausgewählten Stellen des Profils eine Reihe von Bodenproben entnommen worden (Abb.5), von denen sowohl im Anschnitt als auch rechtwinkligen Querschnitt Dünnschliffe hergestellt wurden. Insgesamt betrachtet erbrachten die mikroskopischen Auswertungen eine gute Ergänzung und Bestätigung der im Gelände gewonnenen Resultate, deren Vielfalt am besten mit der Abbildung der Probe 2 (Abb. 6) belegt werden kann. Eindrucksvoll scharf heben sich selbst in der Vergrößerung die Schichtgrenzen ab, die teilweise sogar in Form spaltenartiger Lücken, meistens jedoch durch starke Korngrößenunterschiede unterstrichen werden. Deutlich lassen sich auch die Arbeitsergebnisse im Gelände unterbauen, die die Materialien als lateritische Zerfallsprodukte in verschiedenen Stadien und unterschiedlicher Verteilung in einzelnen Schichten definiert hatten. Die Allgemeingültigkeit dieser Aussagen für sämtliche Proben bestätigt sich schließlich auch in den klar zu beobachtenden Einregelungen der unteren Korngrößen, wie sie beispielsweise am rechten Rand der Abb. 6 zu erkennen sind. Selbst in teilweise vergrößerten Ausschnitten dieser Partien ist noch im Maßstab 1:90 die Orientierung kleinster Teilchen bemerkenswert, die sich auf längere Strecken verfolgen läßt. Derartig beobachtete Anordnungen entsprechen in ihrem jeweiligen Auftreten, innerhalb der einzelnen Proben von verschiedenen Entnahmestellen, genau den angrenzenden, makroskopisch sichtbaren Schichtgrenzverläufen und ihren Verknetungen in allen Lagen und Richtungen. Auffälligerweise sind diese Eigenarten im vorliegenden Untersuchungsgebiet auf ganz bestimmte Korngrößenzusammensetzungen innerhalb der Schichten beschränkt, die sich folgendermaßen eingrenzen lassen: Während absolut keine Einregelungen in Materialien mit geringeren Durchmessern als $0,02 \mathrm{~mm}$ auftreten, ist jenes Kennzeichen am ausgeprägtesten von diesem Grenzwert an aufwärts bis zu Korngrößen von $1,5 \mathrm{~mm}$ zu finden und bestimmt zugleich in jedem Fall für alle ein- und angelagerten größeren Partikel bis zu Kiesgröße 
ihre im Gelände kartierte auffällige Ausrichtung. Demgegenüber lassen die in den vorwiegend jüngeren Schichten auftretenden übrigen Sortierungsgrade von Grobsand aufwärts nur selten vergleichbare Eigentümlichkeiten erkennen; meistens kommt es in jenen Zonen in günstigen Fällen zu parallelen Anlagerungen einzelner Körner unterhalb größerer Kiese, die nur durch eine abwärtige Bewegung jener schwereren Partikel erklärt werden können.

Diese ergänzenden Detailstudien erlauben in der Verknüpfung mit den vorangegangenen Untersuchungsverfahren die abschließende Einengung der gesuchten Verwürgungsursachen der Schichten, von denen die anfangs geäußerte denkbare Möglichkeit durch Hackbau bereits ausgeschieden ist. Auch die von BrEMER (1965) zusammengefaßte Deutung der Gilgaibildungen ist nicht als zutreffender Vergleich anzuwenden. Gleichfalls sind alle Ergebnisse mit einem weiteren vorstellbaren Zusammenhang unvereinbar, der die beobachteten Tatsachen als Folge von Einsinkprozessen spezifisch schwererer Sedimente in unterlagernde Schichten bei völliger Wasserdurchtränkung des Bodens in Erwägung ziehen würde. Zwar haben Butrym u. a. (1964) die dabei selten mehr als zwei Dezimeter erreichenden Bodenbewegungen beschrieben und mit solchen periglazialen Ursprungs verglichen, doch führten diese Umstände nie zu totalen Verwürgungen wie im vorliegenden Untersuchungsbeispiel. Der hier entwickelte Austausch ganzer Schichtfetzen über relativ große Distanzen sowie die meist deutlich erhaltenen Abgrenzungen verschiedener Lagen untereinander lassen sich kaum bei völliger Wasserübersättigung erklären; vor allem würden die beobachteten Einregelungserscheinungen unverständlich bleiben.

Alle gesammelten Beobachtungen und Ergebnisse lassen nur die letzte Erklärungsmög.. lichkeit der fraglichen Bildungen durch Frosteinwirkungen offen, deren Ansätze und Entstehungsursachen sich aus den Aufschlußverhältnissen selbst ergeben. Zwar bestehen im rein äußerlichen Erscheinungsbild auffällige Ahnlichkeiten mit vielen Beispielen aus der umfassenden Literatur, doch gelten diese Umstände allein nicht als Beweis. Offensichtlich liegt der auslösende Faktor der Verwürgungen in den wenigen, auf die Muldenform beschränkten Schichten begründet, die im vergleichsweise ungestörten Normalprofil der Umgebung nicht auftreten. Damit ist bereits der wichtigste Ausgangspunkt gefunden, der die Suche nach den ursächlichen Zusammenhängen auf eine ganz bestimmte verbleibende Anzahl von Schichten und ihre Beschaffenheit konzentriert. Von diesen läßt sich bereits durch das angewandte Rekonstruktionsverfahren die drittoberste Lage abtrennen, die an den umrahmenden Muldenrändern keine Verformungstendenz zeigt (Abb. 5) und deren frühere horizontale Verbindung zur Mitte hin durch Ausmessung kontrollierbar war. Jene Eigenschaften belegen eindeutig nur eine passive Reaktion dieser Zone auf gewisse Beanspruchungen, die teilweise auch im Anschnitt ihres tiefer gelegenen Äquivalents ähnlicher Materialbeschaffenheit sichtbar ist. Das gleiche gilt auch für die begleitenden, mehrfach auftretenden grobkörnigen Ablagerungen, sowohl direkt unterhalb der Oberfläche als auch besonders am Nordrand der Senke, die ebenfalls erst mit zunehmender Annäherung zur Mitte von Verwürgungen und Verknetungen gekennzeichnet sind. Diese übrigens auch in der untersten, ältesten Lage zu beobachtenden Kräfteauswirkungen auf ihre Verformung können schließlich nur den Ausgang von den zwei verbleibenden, aus Feinmaterialien bestehenden Schichten genommen haben, die auch die auffälligsten Veränderungen aufweisen.

An ihr Vorhandensein, ihre beschriebene Zusammensetzung und ihre Lage im gesamten übrigen Ablagerungsverband knüpft sich schließlich die Erklärung und der Beweis für den durch Frosteinwirkung entstandenen Würgeboden (STEEger 1944). Unter der Voraussetzung starker Bodendurchfeuchtung und kalter klimatischer Bedingungen gingen von der Eigenschaft dieser Schichten, der Anziehung erheblicher Wassermengen des Untergrundes während des Gefrierens und der damit verbundenen Ausdehnung, die entscheidenden Wirkungen auf die festgestellten Verformungsprozesse aus. Gerade innerhalb der auf engem Raum stark wechselnden Lagen unterschiedlichster Korngrößen waren die fein- 
körnigen, frostgefährlichen Bodenarten zur Ausbildung von Eisschichtnetzen prädestiniert, während es beim Gefriervorgang innerhalb der gröberen Materialien höchstens zur Eisausfüllung der vorhandenen Porenräume kommen konnte (DüCKER 1954).

Deutlich gehen die Auswirkungen dieser prinzipiellen Entwicklungstendenzen aus dem abgebildeten Profil hervor, selbst wenn es augenblicklich nicht möglich ist, für jede Einzelform die Entstehungsursache durch Frieren oder Tauen oder einer Verbindung beider Vorgänge zu bestimmen. Gleichfalls ist es mangels vorhandenen Vergleichsmaterials ausgeschlossen, die beobachteten und beschriebenen Kräfteeinflüsse auf einmalige oder häufig sich wiederholende Vorgänge zurückzuführen, so daß schließlich nur das Endresultat jener Wirkungen analysiert werden kann.

Für die gesamte Entwicklung war die bereits mehrfach hervorgehobene Tatsache der verhältnismäßig gering beanspruchten, bis $25 \mathrm{~cm}$ hinabreichenden Schichten von ausschlaggebender Bedeutung, deren kaum veränderte Partien nur mit ihrem gefrorenen $\mathrm{Zu}$ stand während der in tieferen Zonen ablaufenden Bodenbewegungen zu erklären sind. Demzufolge mußten sich zwangsweise in jenem unteren Bereich alle Kräftewirkungen in primär abwärts und seitwärts gerichteter Tendenz entwickeln, wobei ihre Bindung an das Auftreten der beschriebenen Schichten im Profilvergleich und den vorher dargestellten Untersuchungsergebnissen zum Ausdruck kommt. In übereinstimmender Weise nehmen daher sowohl die Verwürgungsbildungen als auch die Beanspruchungen des gefrorenen Oberbodens mit ansteigender Mächtigkeit der frostgefährlichen, feinkörnigen Lagen zum Mittelpunkt hin zu, in dessen Bereich sich folglich die größten Pressungserscheinungen bemerkbar machen. Aus diesen Gründen findet sich hier auch der bereits angeführte Austausch von Schichtteilen oder ihr birnenförmiges Einsinken, ihr Durchstoßen bis in das Liegende und wiederum dessen Aufsteigen bis an die Grenze der gefrorenen obersten Bodendecke. Die Wirkungen dieser nur als Ausgleich- und Ausweichbewegungen zu verstehenden Veränderungen sind ihrem Einfluß entsprechend bis in die girlandenähnlichen Formen der untersten Ablagerungen im Muldentiefsten zu verfolgen, wo sie erst am Übergang zum allseits unterlagernden Material enden. Doch werden diese Bewegungsvorgänge auch im aufgezeigten Zusammenhang der untersuchten Mikrostruktur innerhalb der Schichten nachgezeichnet, von denen folgerichtigerweise die frostgefährlichen Zonen mit Korngrößen unter 0,02 mm keine Besonderheiten aufweisen. Dagegen tritt das aufschlußreiche Merkmal der Orientierung von Partikeln größeren Durchmessers bis 1,5 mm und teils darüber in den meisten umgebenden Lagen auf, in welchen infolge der Druck- und Pressungserscheinungen alle Einregelungen den stattgefundenen Fließbewegungen entsprechend ausgerichtet sind.

Alle diese beschriebenen Eigenarten sind typisch für gewisse Bodenfrosterscheinungen in Gebieten höherer Breiten, die sich in vielerlei Veröffentlichungen und in einer besonders großen Anzahl ähnlicher Beispiele bei Dücker (1954) und Steeger (1944) finden. In vielfacher Hinsicht zeigen die makroskopisch vergleichbaren Ausgangsbedingungen und Abhängigkeiten auffallend große Übereinstimmungen. Hingegen stehen bei der Anwendung von Dünnschliffen in ähnlichen Zusammenhängen erste Erfahrungen nur über die Mikrostruktur solifluidal bewegten Bodens von BECKMANN (1967) zur Verfügung.

Schwierigkeiten bereitet allerdings, in Verbindung mit den vorangegangenen Gegenüberstellungen, eine Antwort auf die klimatischen Voraussetzungen für das im Hochland Angolas entdeckte Untersuchungsbeispiel, dessen Entstehung nach allem bisherigen Wissen nicht auf „periglaziale“ Einwirkungen zurückgeführt werden kann. Auch die gegenwärtigen Bedingungen mit ihren nur gelegentlich auftretenden Nachtfrösten sind als mögliche Erklärung völlig unzureichend, selbst wenn im Raume Silva Porto nach Aussagen von Einwohnern die Temperaturen bis auf $-4^{\circ} \mathrm{C}$ absinken können (Borchert 1963). Die verfügbaren Tatsachen erlauben nur die bereits ausgeführte Ableitung eines kalt-feuchten 
Klimas als Ursache der Bodenfrosterscheinungen, da allein unter diesen Bedingungen sowohl die Verwürgungsbildungen selbst als auch die große Bodenplastizität bei stärkster Feuchtigkeitssättigung der betreffenden Schichten und des Untergrundes erklärbar sind. Auffälligerweise waren genau die gleichen klimatischen Eigenarten im zweiten, vorangegangenen Untersuchungsabschnitt von ausschlaggebender Bedeutung, so daß daraus für den vorliegenden Fall nicht nur eine parallele Bestätigung erwächst, sondern auch die gleiche Schlußfolgerung einer im geringen Maße vorhandenen Vegetation in jener Zeit unterstützt wird.

In Fortsetzung der vergleichenden Betrachtung ergeben sich schließlich auch entsprechende Ubereinstimmungen in bezug auf die relative Altersdatierung, die in den inzwischen noch nicht aufgetretenen Verwitterungserscheinungen den Hinweis einer verhältnismäßig kurz zurückliegenden Entstehungszeit trägt. Diese Bedingungen begünstigen vor allem die Kartierung sämtlicher wichtiger Aufschlußgegebenheiten im Gelände, deren makroskopisch sichtbare Einzelheiten nicht die geringsten Veränderungen oder Zerstörungen aufwiesen. Diese gleichfalls ein geringes Alter belegenden Tatsachen werden zusätzlich durch die Untersuchungen der Mikrostrukturen gestützt, die in ähnlicher Weise zu denselben Schlußfolgerungen führen. Selbst in diesen Korngrößenbereichen finden sich wider Erwarten keine Verlagerungen, die vielleicht in Anbetracht der jährlichen Niederschlagsmenge von $1124 \mathrm{~mm}$ und ihrer Verteilung angenommen werden könnten.

\section{Die Altersstellung der untersuchten Ablagerungen}

Obwohl absolute Datierungen auf Grund fehlender Kriterien nicht auszuführen waren, ließ sich doch aus den vorangegangenen morphologischen Untersuchungsergebnissen ein relativ junges Alter der verschiedenartigen Bildungen folgern. Im Fall der letzten beiden Arbeitskomplexe war darüber hinaus eine gegenüber der Gegenwart kältere Klimaphase die Voraussetzung der abgehandelten Formenentwicklung. Bei aller Vorsicht bietet sich so zunächst auch die Möglichkeit, vorläufig die Entstehung der Steinhorizonte als etwa gleichzeitig anzusetzen. Die genaue Datierung und zeitliche Einordnung jener klimatischen Wirkungen bereitet allerdings gewisse Schwierigkeiten, die zum einen in bis heute völlig fehlenden gleichartigen Arbeiten begründet sind. Zum anderen mangelt es im weiten Raum Angolas an entsprechenden, zuverlässigen Daten der bisher kaum bekannten klimatischen Gegegbenheiten aus jüngster geologischer Vergangenheit. Abgesehen von mehr oder minder spekulativen Vermutungen sind erste sichere diesbezügliche Angaben bisher nur aus der Dundo Region im Nordosten Angolas verfügbar, wo aus datierbaren Ablagerungen in $700-800 \mathrm{~m}$ hoch gelegenen Tälern um Mufo $\left(7^{\circ} 36^{\prime} \mathrm{S} / 21^{\circ} 25^{\prime} \mathrm{E}\right)$ der beginnende Versuch einer Chronologie vom Jüngeren Pleistozän bis heute zusammengestellt wurde (Zinderen-BaKker \& Clark 1962). Diese durch verschiedene, sich ergänzende Arbeitsweisen erlangten Daten bieten offensichtlich ein sehr hohes Maß an Sicherheit, sowohl durch die angewandten Methoden als auch durch ihre Parallelisierbarkeit mit entsprechenden Zeitmarken aus Gebieten des östlichen Afrika in ähnlicher Breitenlage.

Dementsprechend könnte auf Grund des erschlossenen, übereinstimmend relativ geringen Alters der eigenen Untersuchungsbeispiele vorläufig nur das Haupt Gamblian als einzig mögliche Entstehungsphase erschlossen werden. Da ausschließlich aus dieser Zeit, in der Spanne von 30500 und 14503 vor der Gegenwart, entsprechende Daten eines kälteren und feuchteren Klimas aus verschiedenen Gebieten vorliegen, bleibt beim jetzigen Stand des Wissens allein diese Periode als erklärende äußere Bedingung für die untersuchten Bildungen übrig. Im Gegensatz zu einem vorhergehenden, dem gegenwärtigen ähnlichen Klima wirkte sich jene folgende, kühl feuchte Epoche für den Nordosten Angolas in einem recht merklichen Temperaturabfall und einer Veränderung der Pflanzenbedeckung aus. Jene Vegetation ähnelte teilweise den heutigen Beständen in den zwischen 
$13^{\circ}-15^{\circ} \mathrm{S}$ und $1700-2200 \mathrm{~m}$ hoch gelegenen Distriktteilen von Moxico, Bié, Benguela und Huila. Damit ergibt sich ein sicherer Indikator für den festgestellten, offenbar sehr deutlichen klimatischen Wechsel, der einer erhöhten Lage des auf $7^{\circ} 36^{\prime} \mathrm{S}$ gelegenen Geländes um mindestens $500 \mathrm{~m}$ entsprochen haben würde. Selbst wenn damit für die eigenen Untersuchungsgebiete in beinahe zweifacher Entfernung vom Äquator und doppelter Höhe die vollzogenen indirekten Schlüsse einer entsprechenden und gleichzeitigen Vegetationsverminderung nicht weiter präzisierbar sind, so fügen sich doch die Ableitungen der morphologischen Untersuchungen gut in jenes Gesamtbild ein. Vor allem belegen die sich gegenseitig stützenden Ergebnisse eindeutig das Absinken der Temperaturen in Verbindung mit der größeren Feuchtigkeit des damaligen Klimas, dessen Auswirkungen und Eigenarten allerdings noch nicht über die vorliegenden Ausführungen hinaus verallgemeinert werden können.

\section{Schlußbemerkungen}

Die unter den anfangs beschriebenen Umständen gewonnenen, verschiedenartigen Beobachtungen und Resultate zeigen deutlich, daß sie zur Zeit nur als einzelne Anhaltspunkte eines im praktischen Sinne bisher kaum bearbeiteten Problemkreises angesehen werden dürfen. Unzweifelhaft sicher müßten sich jedoch weitere, unter den gleichen klimatischen Bedingungen entstandene Zeugnisse verschiedener Art finden lassen.

In diesen Zusammenhängen nimmt offenbar der Planalto Angolas, besonders mit seinen randlichen Bereichen, eine Schlüsselstellung für weitere Untersuchungen zum vorliegenden Komplex ein, dessen Einzelfragen genau wie die Probleme der Warvenzählung und die der Mikrostruktur der Würgeböden weiterhin verfolgt werden müßten.

Alle bisher verfügbaren Tatsachen sind absolut noch nicht ausreichend genug, um aus ihnen bereits jetzt weitere Folgerungen und großräumige Verknüpfungen herleiten zu können. Die aus dem Fehlen verläßlicher Grundlagen verschiedener Art entstehenden Mängel und Schwierigkeiten bestimmen zum großen Teil, wie in der vorliegenden Arbeit, den Gang aller Untersuchungen und beschränken deren Aussagekraft auf den entsprechenden Standort.

Literaturverzeichnis

BeckmanN, W.: Ein Beitrag zur Kenntnis der Mikrostruktur von solifluidal bewegtem Bodenmaterial in der Sierra Nevada (Andalusien). - Anales de edafologia y agrobiologia, Tomo XXVI, Nums. 1-4, 351-360, Madrid 1967.

Borchert, G.: Südost-Angola. - Hamburger Geogr. Studien, 17, Hamburg 1963.

Borges, A. \& Mouta, F.: Sur l'existence et distribution du Karro dans l'Angola. - Compte rendu XV. Int. Geol.-Kongr. South Africa, 2, 186-209, Pretoria 1929.

Bremer, H.: Musterböden in tropisch-subtropischen Gebieten und Frostmusterböden. - Z. f. Geomorphologie N. F., 9, 222-236, Berlin 1965.

Butrym, J., Cegla, J., Dzulnynski, S. \& Nakonieczny, S.: New Interpretation of "Periglacial Structures". - Folia Quaternaria, 17, 1-34, Krakau 1964.

DüCKer, A.: Die Periglazial-Erscheinungen im holsteinischen Pleistozän. - Göttinger Geogr. Abh., 16, Göttingen 1954.

FölSTER, H.: Morphogenese des südsudanesischen Pediplane. - Z. f. Geomorphologie N. F., 8, 393-423, Berlin 1964.

Jessen, O.: Ein Glazialvorkommen in Mittel-Angola. - Geol. Rdsch., XXXV, 38-41, Stuttgart 1934.

Mouta, F.: Nota Explicativa do Esboço Geologico de Angola. - Junta de Invest. do Ultramar, Lisboa 1954.

Steeger, A.: Diluviale Bodenfrosterscheinungen am Niederrhein. - Geol. Rdsch. XXXIV, $520 \mathrm{ff}$., Stuttgart 1944.

van Zinderen-Bakker, E. M. \& Clark, J. D.: Pleistocene Climates and Cultures in North-Eastern Angola. - Nature, Vol. 196, 639-642, London 1962.

Manuskr. eingeg. 18. 12. 1969.

Anschrift des Verf.: Dr. Frithjof Voss, 2 Hamburg 13, Rothenbaumchaussee 21/23, Institut für Geographie und Wirtschaftsgeographie. 